\title{
Cultura escolar: reflexiones sobre su intervención desde una mirada sociocultural de la psicología educacional
}

\section{School Culture: Reflections on Speaking from a sociocultural view of educational psychology}

\author{
Eduardo Guzmán Utreras ${ }^{1}$ \\ Universidad de Santiago de Chile, Chile
}

(Rec: septiembre de 2015 - Acept: noviembre de 2015)

\begin{abstract}
Resumen
Se discute la importancia de la cultura escolar como elemento principal de intervención educacional a considerar por parte de la psicología educacional, entendida como una disciplina que se desempeña en contextos educativos formales. A partir de una mirada sociocultural de la escuela, de la cultura y de la intervención, se busca sintetizar algunos elementos básicos que se deben tener en cuenta para desarrollar la praxis del/la psicólogo/a en estas materias, dando origen a intervenciones complejas, insertas en un contexto educativo y en relación con todos los actores participantes e integrantes de las propias instituciones, generando así el potencial para desarrollar cambios y transformaciones compartidas entre quienes construyen esta cultura escolar. Los apartados siguientes otorgarán énfasis a tres puntos específicos y tensiones a destacar y trabajar como propuesta: 1) El problema de la cultura escolar y la inclusión del psicólogo educacional; 2) La cultura escolar como objeto de interés a partir de una mirada sociocultural; y su interacción con el curriculum; y 3) La descripción de algunos lineamientos básicos orientadores para la intervención a partir de la cultura escolar, desde la mirada de un psicólogo educacional centrado en un paradigma cultural.
\end{abstract}

Palabras clave: cultura escolar, psicología educacional, intervención educacional.

\begin{abstract}
The following article discusses the relative importance of school culture as the main element of educational intervention to be considered by the educational psychology, understood in this context as a discipline plays in formal educational settings. From a sociocultural school look, culture and intervention, it seeks to synthesize some basic elements that must be taken into account in developing the practice of psychologists in these areas, giving rise to complex, embedded interventions in a context education and in relation to all participating actors and members of the institutions, thus creating the potential to develop shared changes and transformations between those who build this school culture. The following sections granted emphasize three specific points and tensions highlight and work as proposed: 1) The problem of school culture and the inclusion of educational psychologist; 2) School culture as an object of interest from a sociocultural look; and their interaction with the curriculum; and 3) a description of some basic guidelines for intervention counselors from the school culture, from the perspective of an educational psychologist focused on a cultural paradigm.
\end{abstract}

Keywords: school culture, educational psychology, educational intervention.

\footnotetext{
1 Correspondencia a: Eduardo Guzmán Utreras. Universidad de Santiago de Chile, Escuela de Psicología, Av. Ecuador \#3650, Estación Central, Región Metropolitana de Santiago, Chile. E-mail: eduardo.guzman@usach.cl
} 


\section{Psicología en la educación y psicología educacional: tensiones frente a la cultura escolar}

Durante los últimos años hemos sido testigos de la gran cantidad de demandas transformadoras de los sistemas educativos, señalando la necesidad de contar con una educación que apunte a la calidad. Para esto se han movilizado recursos humanos y materiales, creándose leyes que buscan, al menos, instalar elementos normativos reguladores que aseguren el cumplimiento de estándares mínimos enfocados hacia lo que se ha intentado denominar calidad en la educación. Bajo estas políticas es que los diversos actores han debido ajustar sus prácticas en virtud del cumplimiento de estos cambios.

Sin embargo, estos cambios estructurales tan sólo aparecen como normativos, es decir, orientan las prácticas del deber hacer en los sistemas educativos en un nivel general y universal. En este sentido, no instalan una base que permita estructurar la posibilidad de ejercer líneas socioculturales en la educación, sino que éstas emergen tan sólo a partir de buenas prácticas flexibles de las escuelas como instituciones formales. La interacción entre esta posición simplista y aquellas posiciones más complejas que emergen inevitablemente de la interacción y construcción social de los actores educativos da origen a elementos como la cultura escolar. Este concepto resulta de suma utilidad al pensar en la intervención educativa como espacio pedagógico de cambio y transformación educacional no ligada a las normativas preferentemente rígidas del curriculum, sino más bien a lo que se le escapa y se considera como transversalidad, objetivos transversales o similares.

En este escenario, es posible apreciar un divorcio entre los lineamientos generales, que promueven explícita o tácitamente las prácticas de enseñanza esperables de los actores involucrados en los sistemas formales de educación, y aquellos que se hacen explícitos por medio de la construcción social que realizan estos actores, como es el caso de los docentes. Surge, pues, una de las tantas tensiones a destacar en esta interacción cuando la construcción cultural se hace permanente y asigna a los actores roles que han sido definidos a partir de representaciones constituidas en otros contextos no educativos, tal es el caso de la psicología.

No es desconocido que para la psicología de la educación el principal centro de interés son los procesos de aprendizaje a partir de su génesis, desarrollo, facilitación y consecución. No obstante, la forma en la cual ha sido entendida esta "ecuación" no siempre ha concentrado los esfuerzos hacia una relación situada, sino más bien hacia una posición excesivamente simplificadora y reduccionista. Así sucede con el enfoque de Edward Thorndike en tanto que el sujeto, el individuo y sus diferencias interindividuales constituyen el centro de estudio y por tanto de praxis.

Frente a esta radiografía, es posible pensar en un rol asignado a la psicología como parte de la cultura escolar imperante y dominante, pero al mismo tiempo fuera de ella. A ésta la llamaremos, convenientemente, psicología en la educación, dado que desarrolla sus prácticas de intervención a través del cumplimiento indirecto de las normativas de la educación formal, utilizando para esto los supuestos externos de la psicología clínica y actuando mediante la lógica terapéutica individual. No obstante, no es objetivo de este escrito detenernos en semejante materia, que ha sido ampliamente discutida en la literatura con el fin de analizar el rol del psicólogo educacional ${ }^{2}$, sino tomar posición a partir de la psicología cultural -educativa- y discutir la importancia de la intervención educacional en la cultura escolar en tanto el individuo y la cultura son inseparables.

Considerar la cultura escolar como objeto principal de interés y consideración implica entenderla como un contexto donde se movilizan los sujetos pedagógicos. Para ello resulta preciso comprender el carácter dinámico de la institución escolar, que construye una realidad histórica y contingente particular a través de la interacción entre sus miembros. Finalmente, esto supone una consideración cuidadosa del concepto cultura en tanto resulta más complejo que en aquella definición a partir del paradigma de la simplicidad. Comprender la intervención educacional a partir del paradigma de la complejidad se ajusta convenientemente a lo que llamaremos psicología educacional ${ }^{3}$, entendiendo que su praxis se hace parte de la comunidad educativa o escolar como una construcción con significados compartidos, supuestos, artefactos, etc. La psicología educacional participa como agente conocedora de la realidad e inserta no tan sólo en la individualidad y la atomización de las tensiones, sino como parte activa del curriculum y la enseñanza como estructura pedagógica, con un sentido construido de lo que significa la educación en

\footnotetext{
2 Algunos autores que han trabajado el tema en Chile son: Baltar, 2003; Baltar \& Carrasco, 2013; Ossa, 2012; Vidal, 2007, entre otros.

3 La APA considera una separación conceptual y práctica entre Psicología Educacional y Psicología Escolar, en tanto sus focos de acción, eminentemente prácticos, se asocian a líneas divergentes desde contextos generales de educación, en el primer caso, y la escuela definida tradicionalmente, en el segundo caso.
} 
sí misma y la formación integral de los individuos que la reciben (Acosta, 2012).

Entender la psicología educacional como una disciplina que se ocupa de la cultura escolar supone enfrentar el reto de la transformación misma de la psicología a partir de sus propias construcciones culturales como disciplina externa a la educación y situada principalmente en un énfasis clínico. Asimismo, implica intervenir en las representaciones sociales que se tienen respecto del rol del psicólogo en la educación por parte de los actores no relacionados con ella directamente. Entraña la transformación del profesional y sus creencias asociadas a la tradición de la psicología, otorgándole de esta forma un lugar justo a dichas prácticas. Supone también construir la praxis a partir de la cultura escolar y de cómo ésta desarrolla sus prácticas educativas. Finalmente, conlleva comprender que la psicología educacional debe asumir la pedagogía como parte de su caracterización y su significado, en tanto ciencia de la educación con características técnicas y reflexivas, dinámicas y creativas. En consecuencia, asumir una mirada compleja de la educación y del rol que le corresponde cumplir como parte de ella.

\section{Cultura escolar}

Los estudios de la cultura escolar han tenido un fuerte desarrollo a partir del siglo XX en la medida que las concepciones de lo educativo han transitado desde la modernidad hacia la contemporaneidad, intentando comprender y explicar los fenómenos educacionales a partir de la mirada interna de quienes los desarrollan. A medida que se avanza desde un paradigma de la simplicidad hacia la complejidad, se ha dejado atrás la visión reduccionista de lo racional, lo interno, el innatismo y la individualidad (Guitart, 2008), de la razón descontextualizada (Bakhtin, 1981, como se citó en Lacasa, 1994), y se ha asumido la posibilidad de un intercambio de símbolos e influencia recíproca de los elementos culturales como la reflexión global y contextualizada de la racionalidad en paralelo con los procesos afectivos y emocionales que dirigen la conducta humana.

En palabras de Roheim (1943), no existe hombre sin cultura y al margen de ésta. Por otra parte, Güell (2008) señala que la cultura es uno de los conceptos más problemáticos en la búsqueda de su operacionalización científica moderna. Citando a Luhmann (1995), Güell indica que la cultura es probablemente "uno de los peores conceptos que alguna vez se haya creado" (p. 38).
La palabra cultura, según la RAE, proviene de "cultivar". Para Benhabib (2006) el concepto procede de la raíz latina colare que se asocia a actividades de preservación, atención y cuidado. Con el tiempo, el concepto fue modificado y alterado por efecto de la modernidad occidental y el pensamiento científico, ajustándose a denominaciones provenientes del romanticismo alemán, a través de las cuales se llamó kultur a una representación de "valores, significados, signos lingüísticos y símbolos compartidos por un pueblo, en sí mismo considerado una entidad unificada y homogénea” (p. 22). Jordán (1997) sintetizó el concepto como un conjunto de significados que otorgan un sentido en la forma de entender la realidad física, social y espiritual de un contexto donde se inserta. Para Cole (1996) refiere a cómo los individuos insertos en una sociedad cultural particular, entretejen conjuntamente acciones sociales entre ellos y las demás cosas que los rodean, sean éstas símbolos, objetos y otros sujetos constructores de cultura. Para Sáez (2006), constituye aprendizaje en tanto involucra costumbres, representaciones sociales, creencias, mitos, valores y rituales no pertenecientes a la herencia genética ni producida por aspectos asociados a ésta. En síntesis, una gran cantidad de autores, han intentado aproximarse a este concepto, por tanto, sería imposible en este artículo ahondar en todos ellos.

De acuerdo a Gálvez (2006), la cultura escolar no tiene una definición propia, clara y consistente, sino que más bien rescata desde la organización su dimensión conceptual como un conjunto de valores, creencias, actitudes, sentimientos y proyectos compartidos por los miembros de una organización (Stolp, 1994). Lobato y Ortiz (2001) la entienden como un conjunto de actitudes, valores y creencias compartidas (contenidos de la cultura escolar) y los modelos de relación y formas de asociación y organización (forma de la cultura escolar) de la escuela (Escobedo, Sales \& Ferrández, 2012). La cultura escolar suele tener en su haber normas y principios, supuestos básicos, ritos, valores y un lenguaje común, que no es solamente propio de una cultura monolítica, sino que también se asocia a una serie de estructuras internas que en conjunto construyen la identidad cultural de una escuela. De igual forma, tales aspectos pueden relacionarse con entidades externas que comparten ciertos elementos de la misma. Esta cultura no se define conceptual ni pragmáticamente como algo alejado de la historia, de la construcción social ni de su contexto; por el contrario, es un concepto histórico, contingente y en constante construcción dinámica, que evoluciona y se modifica a pesar de que en ocasiones 
parezca tener cierta estabilidad; transmitiéndose por medio de elementos formales y definidos por la misma cultura dominante, la cual le otorga sentido y significado (Bolívar, 1996).

De acuerdo a Elías (2015), la realización de múltiples investigaciones en el área de la cultura escolar dio origen a una diversidad de conceptualizaciones, poniendo en riesgo su potencia teórica dada su amplia utilización y poca precisión. Esto probablemente se ajusta al concepto de cultura en sí mismo. Las definiciones dotan de complejidad al concepto y a quien intente relacionarse con él y su aplicabilidad. Referirse a una cultura escolar, éticamente, debería llevarnos a comprenderla desde la complejidad.

El pensamiento complejo responde a una forma de comprensión de la realidad no predecible, en constante construcción, donde el sujeto interacciona con la "realidad" y reflexiona sobre ella, constituyéndose así en una interrelación social, dinámica y transformadora (De Jesús, Andrade, Martínez \& Méndez, 2007). Con certeza, esta mirada que proponemos debe cambiar, éticamente, la forma en la cual la psicología educacional comprende las cuestiones educativas.

Entender a la cultura educativa como una posibilidad concreta, y no tan sólo como un fenómeno emergente de las estructuras formales, extiende los límites marginales y tímidos del objeto de intervención en psicología educativa centrada en la individualidad (Lacasa, 1994). Amplía la mirada hacia elementos que frecuentemente se invisibilizan ante la praxis del/la psicólogo/a en la educación y las prácticas que ejerce dentro de la cultura implícita. Entre ellos, el curriculum y los procesos pedagógicos, de gestión y del entorno que son construidos en la interacción con el contexto y conforman una red de significados compartidos, resistentes al cambio y promotores del desarrollo (Bolívar, 1996).

\section{La relación con el curriculum}

Una de las fuentes más desconocidas y, probablemente, menos consideradas en la praxis de la intervención psicoeducativa por parte de la psicología en la educación se refiere al curriculum, su significado y su pragmática. Autores como Bolívar (1996) y Lacasa (1994), han puesto de manifiesto que la implementación del cambio por medio de la cultura escolar debe considerar aquellos elementos que le son propios en la construcción social de los individuos. Hoyle (1971) afirmó que una de las formas de generar un cambio social era posible por medio de un cambio curricular, en tanto se transforma en un instrumento que permite mediar entre la cultura y la educación (Lacasa, 1994). Un artefacto cultural que representa al mismo tiempo la construcción social realizada en la interacción, por medio de un lenguaje codificado, y que debe ser materializado como un instrumento para su utilización (Cole, 1991).

El curriculum es un instrumento idealista y material que cumple un rol mediador entre la sociedad y la educación, en tanto se materializa como enseñanza a través de los lineamientos que entrega. Es "en el curriculum donde se concretan y toman cuerpo una serie de principios de diversa índole-ideológicos, pedagógicos, psicopedagógicos- que, tomados en su conjunto, muestran la orientación general del sistema educativo" (Coll, 1994, p. 21). Según Bordieu (1989), el curriculum sería un instrumento reproductor de las ideas predominantes en determinada época, y la escuela, aquel lugar donde se puede constituir e instaurar los esquemas de pensamiento que permiten dicha reproducción cultural.

No obstante, en este proceso de mediación se observan elementos que generan tensiones internas, como es el caso de factores políticos, técnicos y globalizadores. El currículum está sometido a una serie de presiones multifocales que responden a lógicas propias de la sociedad en la que vivimos (Magendzo, 2008), y que entran en tensión en momentos de discusión pragmática en donde la globalización y la interculturalidad generan movimientos de apertura de fronteras y producen propuestas inclusivas o de territorialización aferrada a la cultura local (García-Canclini, 1995).

A diferencia de Bordieu, Bernstein (1988) expresa que los elementos fundamentalmente culturales que se intentan transmitir por medio de la construcción de un curriculum no responden necesariamente a la lógica de construcción y reconstrucción efectiva y determinada por la posición social, dado que no se definen los mecanismos por los cuales se transmitirán estos contenidos prescritos. Por tanto, la noción de lo escrito y determinado como contenido educativo se ve influenciada por aquellos instrumentos pedagógicos que buscan materializar el contenido prescrito. Estos responden a posiciones privilegiadas y no privilegiadas desde el punto de vista familiar, social y/o cultural donde se intenta reproducir el contenido. De esta manera, se plantea la existencia de una segunda dimensión del curriculum, aquella que emerge en la práctica, la que se genera en la interacción, en la pedagogía, y que en palabras de Surjo Torres (1991, como se citó en Lacasa, 1994) se le ha llamado curriculum oculto. 
El curriculum oculto responde a las prácticas emergentes, aquellas que no se definen explícitamente, y que emergen por medio de las creencias, concepciones o valores éticos de los pedagogos, sujetos pedagógicos y distintos actores que se involucran en la institución educativa, o bien emergen por medio de las estrategias didácticas involucradas en la bajada curricular, en la gestión, en la interacción, es decir, en llevar a la práctica aquello que nos dice el curriculum.

Estableciendo una analogía entre cultura escolar y curriculum, el punto de convergencia apunta precisamente hacia el curriculum oculto, en tanto se relaciona con esta cultura en la práctica, en lo que Bernstein (1988) llama instrumento pedagógico, en la pedagogía, en la intervención educativa que se genera en el contexto, en la interacción y dentro de los factores involucrados en la definición de cultura escolar.

Cuando se forma parte de la cultura educativa, se comparten ciertas lógicas particulares a ella y, por tanto, se generan cuatro posibilidades emergentes:

1. No se forma parte de ella explícitamente, pero se es sometido implícitamente, reproduciendo así innovaciones sin cambio (Rudduck, 1991, como se citó en Bolívar, 1996), como aparentemente es el caso mayoritario de la psicología en la educación, constituyéndose así el/la psicólogo/a educativo/a en una parte más del eslabón que construye un curriculum oculto reproductor con sus consecuentes prácticas.

2. No se forma parte de ella y tampoco se tienen las herramientas para generar cambios y, por tanto, se genera una autosegregación de la institución al no tener la voluntad de sometimiento a la dominancia; en consecuencia, comúnmente, se genera una alta rotación en el cargo, lo que nuevamente continúa perpetuando una construcción particular de lo oculto y sus respectivas prácticas asociadas.

3. Se tienen herramientas para la intervención cultural, pero al no ser consciente de ella y su complejidad, la identificación y compromiso en el rol se mantiene bajo las lógicas reproductivas de la misma.

4. Se forma parte de ella y se reconoce esta inclusión conscientemente, se tienen conocimientos y competencias reconocidas $\mathrm{y}$, por tanto, se considera la labor del/la psicólogo/a educativo/a como un elemento transformador, ya que esta lógica constituye por sí misma una intervención que prepara las condiciones para el cambio educativo.

El proceso de cambio o adaptación curricular no responde únicamente a modificaciones, aperturas o transformaciones estructurales de la gramática escolar ${ }^{4}$, sino que es un proceso más complejo en donde los cambios se producen en todos los actores involucrados, por medio de decisiones democráticas que instauran las lógicas de transformación educativa en beneficio de la calidad. No bastan las modificaciones estructurales que presionan al currículum explícito, como por ejemplo leyes o reglamentos, sino también la interpretación y aplicación de éstas en la dinámica rutinaria de los actos pedagógicos y en el curriculum oculto como campo de acción potencial de una psicología educacional transformadora de la cultura escolar.

\section{La intervención educativa cultural y su vinculación pedagógica en la psicología educacional}

En la actualidad, aún mantenemos la discusión respecto de cuál es el real significado de la intervención educativa que debe realizar nuestra disciplina, principalmente en los contextos escolares o formales, dado el divorcio entre psicología y pedagogía. Los profesionales de la Educación esperan encontrar en la psicología una orientación y una guía de sus acciones pedagógicas (Coll, 1992), generándose expectativas y objetivos de intervención que no siempre se ajustan a las reales necesidades del sistema escuela, conformando de esta manera, prácticas cruzadas de solicitudes y acciones ejecutadas desde un modo experto. La Psicología en la Educación no ha logrado otorgar respuestas satisfactorias a las problemáticas educativas reales, manteniéndose esclava de conocimientos y procedimientos que culturalmente se asocian a una construcción moderna. Esta busca relacionar desde el modo experto a la ciencia, el conocimiento científico y la técnica, con aquellos sujetos pedagógicos que poseen un "problema" o una "situación problema". Ejemplos de lo anterior se pueden apreciar en todas aquellas ejecuciones profesionales que realizan los psicólogos en la educación en escuelas, por medio de programas de integración o talleres externos focalizados, buscando la solución a problemas innatos, internos, individuales y racionales,

\footnotetext{
${ }_{4}$ A diferencia de la cultura escolar, la gramática escolar fue descrita en Norteamérica y hace referencia a "estructuras, reglas y prácticas que organizan la labor de la instrucción". Ejemplo de esto lo constituyen "prácticas tan familiares como la graduación de los alumnos por edades, la división del conocimiento por materias separadas y el aula autónoma con un solo maestro" (Tyack \& Cuban, 2001, p. 23; como se citó en Elías, 2015).
} 
los que inclusive parecieran ser considerados como naturales ante el diagnóstico nosológico y el tipo de consideración interventiva que se intenta ofrecer y entregar.

No obstante lo anterior, gran parte de las exigencias de la intervención moderna provienen de las mismas exigencias que conocen y eligen los actores educativos, esperando que la psicología les otorgue respuestas, orientaciones y soluciones a partir de un dominio experto puntual. Esta idea resulta fundamental en el análisis de la intervención en la cultura escolar por varios aspectos. En primer lugar, por las altas expectativas que poseen los actores pedagógicos respecto de la intervención educativa por parte de la psicología, modelo y método proveniente desde la ciencia psicológica, y por tanto "certificado" para corregir los errores, simulando forzadamente un modelo que podríamos asociar al "médico". En segundo lugar, está el bajo conocimiento de la psicología en la educación con respecto al concepto mismo de la educación y las formas de control que ejerce por medio de acciones pedagógicas sobre la cultura escolar a través de la enseñanza y la escolarización. Esto es debido su formación excesivamente centrada en aspectos operacionales que provienen principalmente de la clínica. Finalmente, nos encontramos con el alto nivel de fracaso que tendrá la intervención, dada la desconexión entre los significados compartidos, útiles para el cambio de quienes conforman las instituciones educativas; los requerimientos educativos propiamente tales, y los "sujetos problema" que han sido designados y "certificados" por la misma institución en asociación con quienes ésta confía sus problemas educativos.

La cultura escolar emerge entonces como una realidad posible, que permite comprender cómo se articulan y funcionan las instituciones educativas a partir de sus actitudes, valores, creencias, modos de relación, organización y asociación (Escobedo et al., 2012). De esta manera, se le otorga un estatus de psicología educacional, dado que nos obliga a la inclusión de elementos pedagógicos en lo propiamente educativo. Dicho de otro modo, se debe hacer parte de la educación a aquello que por sí mismo interfiere en los procesos educativos, pero que no se considera pedagógicamente abordable por no constituir un objetivo explícito del curriculum formal. Se trata de generar pedagógicamente estrategias de forma tal que dichos fenómenos sean sistematizados y evaluados de igual forma que el contenido curricular designado por las tradiciones cognitivas. Para eso se requiere principalmente de un trabajo transdisciplinario en conformidad con otros actores facilitadores de las escuelas que también ejercen labores pedagógicas en los componentes teórico-prácticos que intervienen en la labor escolar, en la transmisión y construcción de significados compartidos por medio de interacciones de todos los facilitadores o mediadores, sean éstos personal pedagógico, sujetos pedagógicos, personal de acompañamiento educacional ${ }^{5}$ y/o del ámbito familiar. Intervenir desde la cultura escolar obliga al/ la psicólogo/a educacional a conocer, comprender e interrelacionarse con la educación, y no tan sólo con los modelos terapéuticos tradicionales; implica comprender la pedagogía como vehículo al servicio de la explicación, interpretación y transformación de cosas, hechos y acciones educativas (Touriñán, 2011), traduciendo la cultura escolar y articulándola para transformarla en un instrumento susceptible de modificar por medio de procesos educativos.

La intervención educativa, en este sentido, debe promover que la cultura escolar se traduzca en una acción intencional conducente a un desarrollo integral de los sujetos pedagógicos y de quienes conforman las comunidades educativas. En ambos tipos de actores, se ha de tener en cuenta su carácter dinámico, sus propósitos, sentidos, supuestos, ritos instaurados, metas declaradas e implícitas con el objetivo de ejercer acciones que, intencionadamente, generen cambios en la construcción. A modo de ejemplo, en términos prácticos, traducir pedagógicamente el significado que tiene una mala conducta, un informe de calificaciones, un reporte de felicitaciones, los actos cívicos matutinos y las formaciones previas al ingreso al aula (por describir una pequeña porción de cosas dentro de la diversidad de posibilidades) resulta didáctico para comprender a lo que referimos.

La consideración de la cultura escolar como parte de una intervención educacional supone una reorganización de las concepciones sobre la representación del rol que tienen los/as psicólogos/as y sobre la forma en que se ejecutan las acciones al interior de una escuela. Ya no se trata de desarrollar un modelo de réplicas dentro de la escuela; sino de llevar a cabo procesos más complejos de diagnóstico educativo, intervención e investigación aplicada al contexto. También tiene que ver con el establecimiento de un modelo interactivo que siga dinámicamente los lineamientos que la psicología cultural nos ofrece; esto es, entender que los procesos de aprendizaje y de desarrollo humano tienen lugar en la cultura (De la Mata \& Cubero, 2003). La cultura se

\footnotetext{
5 Se prefiere utilizar este término en vez de "asistente de la educación”, dado que se busca dar un sentido de participación activa e interna a quienes no conforman los equipos formales de educación.
} 
entiende a partir de símbolos, significados, prácticas y constructos compartidos (Guitart, 2008), a través de distintas unidades culturales que están relacionadas explícita e implícitamente con la escuela, y que forman parte de ella mediante esta mirada.

La importancia de estas unidades culturales como parte del proceso de aprendizaje y desarrollo de los individuos ha sido desarrollada, principalmente, por Lev Vigotsky y la psicología cultural, pero para estos efectos, depurada por Monereo y Solé (1999). Estos autores han destacado que el desarrollo humano conlleva procesos de culturización donde los niños y niñas se enfrentan a contextos culturalmente organizados y son los adultos quienes seleccionan, interpretan y transforman dicho contexto mediante acciones verbales o no verbales. Es por medio de esta culturización que se produce la apropiación paulatina de elementos que aportan a sus procesos mentales, y son estos últimos los que permiten a cualquier aprendiz apropiarse del mundo exterior e interior (emociones, afectividad, impulsos, deseos, motivaciones), es lo que Vigotsky llamaría conciencia humana.

La interacción entre aquellos llamados "agentes mediadores" (padres, familiares, pedagogos) y el estudiante, siempre se produce en un contexto social en donde los significados son asumidos y compartidos como propios y particulares de ese contexto específico, perdiéndose dichos significados fuera de éste. En el caso específico de la escuela y sus actores, dichos significados no necesariamente se tornan compartidos por todos quienes están insertos en ella (profesores, estudiantes, otros actores), y por tanto, en muchas ocasiones carecen de significado, inclusive dentro de la misma cultura escolar, tornándose ésta difusa y perdiendo potencialidad práctica explicativa.

La escuela, por excelencia, tiene un carácter teleológico y frente a ese carácter requiere precisamente de la pedagogía. No obstante, la bajada curricular que se realiza a través de la pedagogía, genera condiciones propias a partir de los elementos subjetivos de quienes son portadores del conocimiento pedagógico. Estos elementos, por lo demás, son inevitables en los seres humanos y conforman instancias culturales que si bien no constituyen educación en sí mismas, interfieren en sus procesos. La vinculación entonces entre curriculum oculto, pedagogía y cultura escolar parece ser un hecho concreto.

\section{Mediación en la cultura escolar}

Tan complejo como definir cultura y entender la cultura escolar, es también intentar estructurar una lógica de intervención específica en ella. La cultura refiere a supuestos, significados y valores compartidos, prácticas educativas y de enseñanza que históricamente se van instalando en quienes se identifican con la cultura escolar. Siguiendo a Vigotsky (1979), el aprendiz puede acceder a nuevos estadios de competencia a través de la actividad mental constructiva que puede desplegar gracias a un proceso mediado. La mediación se transforma en una interacción adecuada entre los artefactos educativos y la significación que los aprendices internalizan (Cole, 1991) y está construida en un contexto sociocultural que posee significados, valores, prácticas, etc. Entonces, la educación es "una apropiación dotada de sentido y significado en la cual el individuo se reconoce en la interacción como sujeto cultural activo que se recrea con aquello que la cultura le ofrece" (Ilizástigui, 2015, p. 39).

Labarrere (2008) ha argumentado que a nivel de proceso, el fenómeno de la mediación tiende a tornarse invisible para el aprendiz, produciéndose la pérdida de involucración por parte del mismo como actor activo en su proceso de desarrollo, no teniendo acceso a los mecanismos que lo produce. La conciencia institucional educativa ha de ser internalizada explícitamente en los elementos de la cultura escolar y traducirse pedagógicamente en prácticas conscientes en relación a los objetivos de la mediación pedagógica que realiza, desde ahora, "el o la interventora". Vislumbramos entonces una forma de entender el rol del/a psicólogo/a educacional, como mediador/a en la cultura escolar, y un nuevo dominio teórico-práctico de desarrollo profesional: la pedagogía.

La pedagogía como tal resulta un concepto tan complejo como el de cultura escolar. No obstante, considerarla en su magnitud o concepción técnica nos parece de suma relevancia como un acto del hacer y una forma compleja de entender la intervención educativa al tener un objetivo, método, planificación y reflexión de base acerca de lo que se requiere en un contexto educativo (Luzuriaga, 1981). Touriñán (2011) describe la intervención pedagógica como una tarea educativa intencionada con, por y para el educando, justificándose en el conocimiento y funcionamiento 
del sistema educativo. Asimismo, la intervención educativa es descrita como una acción intencional para la realización de prácticas intencionadas conducentes a un desarrollo integral, pudiendo ser formal, no formal o informal, pero siempre respetando el carácter agente del educando (Touriñán, 2011).

Entender que la pedagogía es una técnica reflexiva ${ }^{6}$ con conocimiento de la educación y relacionada específicamente con la intervención educacional, supone un reto para la formación en la psicología educacional, en tanto enfrentamos la obligación de educar para el cambio sociocultural en las instituciones. Lograr esto supone entender que la escuela es una institución donde históricamente se generan, transmiten y reproducen los elementos culturales, donde el diagnóstico educacional puede vislumbrar problemáticas que actúen como barreras al desarrollo y aprendizaje de la escuela ${ }^{7}$, y en donde dicha realidad debe ser transformada por medio de la pedagogía como técnica reflexiva mediadora entre la realidad contextual y las posibilidades que ésta tiene conscientemente en virtud de su desarrollo y mejora de los procesos educativos. De este modo, si no se conoce la potencialidad para mediar estos cambios, la escuela se mantendrá estática en su capacidad transformadora y su potencialidad nunca será suficientemente explotada (Martínez-Otero, 2003).

Para desarrollar una propuesta de intervención en la cultura escolar se debe partir de una premisa fundamental: toda intervención debe ser constante, en tanto las instituciones se van construyendo en la interacción, y no tiene un principio ni un fin naturalizado. Esta premisa ayuda a trascender las figuras que determinan un tiempo de inicio y fin. Junto a la continuidad o fracaso de las propuestas de la acción está el acompañamiento ${ }^{8}$ desde la posición de un facilitador activo y participante de las propuestas que se construyen conscientemente en la institución educativa. El cambio cultural implica la transformación de la propia identidad, por lo que no puede ser gestionada sino reconstruida (Bolívar, 1996), a partir de la capacidad,

\footnotetext{
6 Entendemos que la pedagogía debe ser considerada como una disciplina que va mucho más allá de la mera simplicidad de su consideración técnica, exclusivamente. Más bien, ha de ser situada en una discusión que la señale como parte imprescindible de las ciencias de la educación.

7 En temáticas como la modificación de las prácticas pedagógicas, la inclusión educativa, la interculturalidad, por describir algunas.

8 En algunas áreas sociales, el acompañamiento es una estrategia de acción similar a lo que comúnmente se conoce como intervención, constituyendo su diferencia en la conexión y relación con la realidad social, y no como impuesta externamente.
}

el compromiso, la motivación, las creencias, etc. Fullan (1993) indica que la resistencia al cambio no es tan compleja, sino más bien la presencia de innovaciones que han sido adaptadas acríticamente y de forma superficial, no considerando esta propia identidad.

El proceso de reconstrucción de la cultura escolar supone un aprendizaje que debe seguir la lógica de la intermediación, buscando intervenir no tan sólo en los significantes construidos en un plano abstracto, sino también a través de prácticas concretas que son modeladas y reforzadas en la realidad cotidiana. En completo acuerdo con Labarrere (2008), se trata de elementos simbólicos y, adicionalmente, de elementos en la práctica humana por medio del modelaje. Esto resulta de tremenda importancia frente a las acciones pedagógicas que debe realizar el/la psicólogo/a educacional en su actuar, dado que será necesario articular estos elementos abstractos y subjetivos de la cultura escolar (supuestos, creencias, concepciones y otros) con lo abstracto de los significados que se generan en la interacción escolar (significados colectivos, compartidos y construidos en las prácticas) de los miembros y/o actores educativos. Adicionalmente, también se requerirá instrumentalizar convenientemente las acciones concretas suficientes en relación con los objetivos de la intervención. Cabe señalar que estos objetivos surgen del diagnóstico construido en la cultura misma y deben transitar hacia acciones lógicas mediadoras, susceptibles de ser apropiadas por los integrantes de la cultura tanto abstractamente como a través de hechos que los mismos integrantes van moldeando, como producto concreto de la acción pedagógica transformadora.

\section{Orientaciones para la intervención en la cultura escolar}

Hasta acá hemos discutido algunas ideas a partir de definiciones conceptuales de la cultura escolar y su interacción con la psicología educacional y la pedagogía. Pretender cubrir todas las aristas involucradas en estas definiciones y posibilidades teórico-prácticas sería imposible. No obstante, los planteamientos descritos nos permiten exponer algunas orientaciones fundamentales respecto de la intervención educacional en la cultura escolar. En concreto, no buscamos construir manuales en los cuales nos aventuremos a sugerir cómo se realiza la intervención educacional en la cultura. Sin embargo, resulta fundamental sistematizar, siguiendo el ejemplo pedagógico al cual hemos adscrito, algunos 
puntos mínimos a considerar frente a la labor del/la psicólogo/a educacional como interventor o acompañador de la cultura escolar. A continuación se procederá a su descripción.

Resulta fundamental comprender que la intervención educativa es un proceso que comienza cuando se decide intervenir en relación a una situación contingente que emerge como posibilidad de cambio (se identifican tensiones internas, conveniencias, etc., y se levantan antecedentes junto a una justificación de la intervención, similar a un diseño científico propiamente como tal). A raíz de lo anterior, afirmamos que la cultura escolar no necesariamente es un problema, sino más bien es una potencialidad en el sentido estricto de la mejora. Las posibilidades nos llevan a mejorar la situación actual por medio de las potencialidades de la escuela misma y nos alejan de una concepción del déficit tan abusada por la psicología en la educación.

La apropiación y conocimiento de la cultura escolar implica desplegar una gran cantidad de recursos de concientización de ésta, descartando aspectos monolíticos y simplistas, y comprendiéndola a partir de la complejidad. En ella conviven una serie de actores que la construyen, pero no necesariamente actúan como un sólo grupo con creencias o supuestos únicos e incuestionables, sino cada cual desde su originalidad, individualidad y el rol que ejerce.

El conocimiento de la cultura escolar supone la interacción con los individuos constitutivos de ésta: directivos, docentes, técnicos, estudiantes, familias, acompañantes de la educación; pero a la vez, también supone los artefactos construidos propios del contexto sociocultural (libros, decretos, declaraciones de intención educativas, programas educativos institucionales, etc.).

La escuela se hace en la interacción por quienes la constituyen y por las demandas externas que se hacen en relación a su significado social. Esta interacción provoca hechos que no siempre son conscientes en la representación que tienen los actores respecto de sí mismos y su cultura escolar. Por tanto, el proceso de cambio funciona a una velocidad no determinada externamente por artefactos impuestos, tampoco por la opinión de expertos ajenos, sino en función de su identificación interna y sus lógicas de funcionamiento.

Principalmente, son las necesidades del curriculum prescrito las prioridades de trabajo de la escuela. No obstante, entender la cultura escolar no significa trabajar necesariamente en estos temas explícitos, sino más bien de abrir el campo a aquellos que resultan desconocidos, que no se hacen conscientes para quienes conforman esta cultura, pero que se presentan en la realidad de su experiencia.

La identificación de las tensiones y su potencialidad da lugar a un diseño metodológico o acción pedagógica, que conlleva objetivos plausibles y marcos de acciones concretas pero al mismo tiempo flexibles, que permiten desarrollar procesos mediadores de cambio en toda la escuela y no sólo en individuos aislados. En este sentido, resulta útil plantearse las preguntas clásicas que organizan el curriculum: ¿qué?, ¿cuándo?, ¿para qué?, ¿cómo evaluar?, ¿quién o quiénes?, entre otras. Los hechos están ahí, no se deben inventar.

Las estrategias pedagógicas del/la psicólogo/a deben considerar la resignificación simbólica de la cultura escolar, asunto de muy difícil modificación si se trabaja en abstracto, y además, la mediación de prácticas modeladas en forma concreta para acceder a la conciencia de la mayor parte de actores involucrados y hacerlos partícipes de la reconstrucción de la misma. La estrategia de cambio debe desarrollar una unión de prioridades y deliberar ciertas actividades que no funcionen de un modo gerencial, sino colaborativo (Bolívar, 1996). Estas estrategias pedagógicas deben ser consecuentes con la definición misma del término y su aplicación, es decir, deben contemplar espacios, tiempos, materiales necesarios, etc.

Por otro lado, desde el sentido común se tiende a pensar que la evaluación debe formar parte del fin de la intervención, lo que constituye un grueso error en este contexto. La evaluación debe comenzar junto con la intervención misma, en tanto acompaña diagnósticos iniciales, de proceso y finales, aportando información respecto de los contenidos agentes de la cultura escolar y las posibilidades de flexibilidad frente a la metódica definida en nuestra estrategia pedagógica inicial. Este es un punto delicado que, si bien no trataremos en detalle acá, debe ser destacado, ya que una de las grandes amenazas al cambio podría generarse en la incertidumbre sobre la frase "para qué evalúan mi trabajo". Así, se podría asumir entonces una falta de conexión con el contexto y una sensación de externalidad que provoca una resistencia al cambio e incluso, el levantamiento de barreras de protección de la misma cultura escolar. De esta forma, se relega al/la psicólogo/a educacional a una condición de psicólogo en la educación, situación que no tan sólo aqueja a esta área, sino a todos quienes no sean considerados parte de la identidad de la cultura en cuestión.

En resumen, la intervención educativa en la cultura escolar resulta una actividad compleja pero holística. Busca generar elementos que permiten pensar en un 
cambio significativo de los supuestos, valores, ritos y prácticas, que forman parte de la identificación de las instituciones educativas, yendo más allá del mero asistencialismo reactivo, de la fragmentación acotada de los fenómenos contextuales educacionales y más allá de los cuatro reduccionismos a los cuales refiere la psicología cultural.

Intentar dar respuesta íntegra a temas como la cultura sería prácticamente idealista e imposible; asimismo, intentar capturar lo esencial, la realidad de la cultura escolar y al mismo tiempo de la psicología educacional sería un despropósito de nuestra parte, finalizando en el fracaso como tantas intervenciones educacionales realizadas bajo la lógica reduccionista y descontextualizada. Esta analogía tiene un fundamento común: en ambos casos no se describe y asume la realidad del tema a partir de su complejidad. En consecuencia, arriesgarse a consolidar una respuesta definitoria y final conduce a la resistencia y a la desconsideración de lo que, impetuosamente, queremos realizar, responder y transmitir como intervención en estas materias.

Trabajar con la cultura escolar conlleva una serie de retos y desafíos para la psicología educacional en tanto ésta se entienda como parte de las intervenciones educativas, y no como responsable absoluta y personal de ellas. La formación de profesionales que desarrollarán labores en el área educativa formal, no formal o informal debe apuntar en una medida justa al desarrollo de competencias para la intervención en sistemas escolares complejos, más allá del asistencialismo clínico, del cual no renegamos, sino que más bien ampliamos a partir de la complejidad misma de los procesos educativos. En el caso de ejemplos concretos como la interculturalidad, la diversidad, las necesidades especiales de enseñanza, convivencia escolar, burnout, etc., la mera intervención focalizada en síntomas o individuos aislados podría garantizar un juicio clínico y una mejora terapéutica bajo esas lógicas. Sin embargo, este tipo de intervención no necesariamente garantiza que el aislamiento se reproduzca en un sistema que en sí mismo no sea capaz de elaborar estrategias de rearmado interno y aceptación, buscando mantener una estabilidad y equilibrio interno, y haciendo frente a la perturbación que supone la intervención educativa (Monereo \& Solé, 1999).

Finalmente, conviene realizarse una pregunta que hemos circundado implícitamente y que otros autores también se han planteado de la misma forma con anterioridad (Gálvez, 2006; Martínez-Otero, 2003): ¿En qué medida la educación es capaz de ofrecer lo que los individuos miembros de una sociedad o cultura poseen como expectativas o motivaciones? No siempre la escuela se corresponde con lo que la sociedad desarrolla o construye. Por tanto, aquellos elementos internos construidos históricamente, y que se mantienen por medio de supuestos, valores, ritos y prácticas, no se corresponden con la realidad propiamente como tal, provocándose tensiones internas en la interacción sociocultural. Al desempeñarse en el campo de acción de lo educativo sin verse directamente involucrada en el curriculum oficial, la psicología educacional se sitúa en un lugar privilegiado, permitiéndole apreciar la educación, la enseñanza y la escuela misma en forma externa a la cultura, pero siendo participante de ésta. Entonces, resulta muy favorable actuar desde estas posibilidades complejas de intervención y apropiarse de la pedagogía, como herramienta portadora de la educación, para ser parte del sistema e intervenir desde la mediación sociocultural en virtud de las mejoras.

Bruner (1990) afirmó que la cultura moldea la vida y la mente humana. Vigotsky indicó que la relación entre el desarrollo individual y social se da dialécticamente, considerando un "determinismo" socio-histórico del desarrollo; entonces la dimensión del individuo está determinada por su existencia social y cambia a la par de las modificaciones contextuales.

\section{Referencias}

Acosta, F. (2012). Educar, enseñar, escolarizar: el problema de la especificación en el devenir de la pedagogía (y la transmisión). Tendencias pedagógicas, 20(1), 93-105. Recuperado de http://www.tendenciaspedagogicas.com/Articulos/2012_20_07.pdf

Baltar, M. (2003). Asignatura Pendiente del Psicólogo Educacional. En E. Neuman, (Ed.), Campos de Interferencia, Subjetividad e Institución (pp. 183-203). Santiago: Publicaciones Universidad Arcis.

Baltar M. \& Carrasco C. (2013). Re-pensando la psicología educacional en Chile: Análisis crítico de su quehacer y sugerencias proyectadas. Psicología para América Latina, 24, 173-190. Recuperado de http://www.revistapsicolatina.org/ wp-content/uploads/2013/08/Revista_24.pdf

Benhabib, S. (2006). Las reivindicaciones de la cultura. Igualdad y diversidad en la era global. Buenos Aires: Katz.

Bernstein, B. (1988). Poder, educación y conciencia. Sociología de la Transmisión cultural. Santiago de Chile: CIDE.

Bolívar, A. (1996). Cultura escolar y cambio curricular. Bordón, 48(2), 169-177. Recuperado de http://www.icshu.net/downloads/ microtalleres/ej_Cultura\%20Escolar\%20y\%20Cambio\%20 Curricular.pdf

Bordieu, P. (1989). Sistemas de enseñanza y sistemas de pensamiento. En Gimeno-Sacristán, J. \& Gómez A. (Eds), La enseñanza: su teoría y su práctica (pp. 20-36). Madrid: Akal.

Bruner, J. (1990). Acts of Meaning. London: Harvard University Press. 
Cole, M. (1991). A cultural theory of development: what does it imply about the application of scientific research. Learning and Instruction, 1(3), 187-200. doi:10.1016/0959-4752(91)90002-P

Cole, M. (1996). Cultural Psychology: A Once and Future Discipline. London: Harvard University Press.

Coll, C. (1992). Constructivismo e intervención educativa. ¿Cómo enseñar lo que ha de construirse?. Aula de innovación educativa, 2 , 79-82. Recuperado de http://medicina.iztacala.unam.mx/medicina/ Coll\%201992.pdf

Coll, C. (1994). Psicología y curriculum. Buenos Aires: Paidós.

De Jesús, M., Andrade, R., Martínez D. \& Méndez, R. (2007). Re-pensando la educación desde la complejidad. Polis Revista Latinoamericana, 16, 2-13. doi:10.4000/polis.4581

De la Mata, M. \& Cubero, M. (2003). Psicología Cultural: aproximaciones al estudio de la relación entre mente y cultura. Infancia y Aprendizaje, 26(2), 181-199. doi:10.1174/021037003321827777

Elías, M. (2015). La cultura escolar: un concepto complejo. Educare, 19(2), 285-301. doi:10.15359/ree.19-2.16

Escobedo P., Sales, A. \& Ferrández, R. (2012). La cultura escolar en el cambio hacia la escuela intercultural inclusiva. Edetania, 41, 163-175. Recuperado de dialnet.unirioja.es/descarga/articulo/4089718.pdf

Fullan, M. (1993). El significado del cambio educativo: un cuarto de siglo de aprendizaje. Revista de currículum y formación del profesorado, 6(1-2), 1-14. Recuperado de https://www.ugr. es/ recfpro/rev61ART1.pdf

Gálvez, A. (2006). Motivación hacia el estudio y la cultura escolar: Estado de la cuestión. Pensamiento psicológico, 2(6), 87-101. Recuperado de http://revistas.javerianacali.edu.co/index.php/ pensamientopsicologico/article/view/51/150

García-Canclini, N. (1995). Consumidores y ciudadanos, conflictos multiculturales de la globalización. México: Grijalbo.

Güell, P. (2008). ¿Qué se dice cuando se dice cultura? Notas sobre el nombre de un problema. Revista de sociología, 22, 37-64. doi:10.5354/0716-632X.2008.14476

Guitart, M. (2008). Hacia una psicología cultural. Origen, desarrollo y perspectivas. Fundamentos en Humanidades, 9(2), 7-23. Recuperado de http://fundamentos.unsl.edu.ar/ pdf/revista-18.pdf

Hoyle, E. (1971). How does the curriculum change?. En R. Hooper (Ed.), The curriculum: context, design and development (pp. 375398). Edinburg: The Open University Press.

Ilizástigui, L. (2015). El modelo de intermediación en la evaluación cognitiva de niños. Madrid: Dykinson.

Jordán, J. (1997). Propuestas de educación intercultural para profesores. Barcelona: Ceac.
Labarrere, A. (2008). Bases conceptuales de la mediación y su importancia actual en la práctica pedagógica. Revista Summa Psicológica UST, 5(2), 87-96. doi:10.18774/summa-vol5.num2-214

Lacasa, P. (1994). Aprender en la escuela, aprender en la calle. Madrid: Visor.

Lobato, X. \& Ortiz, C. (2001). La importancia de la cultura escolar para la Escuela inclusiva. En A. Coruña (Ed), Atención educativa a la diversidad en el nuevo milenio: XVIII Jornadas de Universidades y Educación Especial (pp. 677-684). España: Universidade da Coruña.

Luzuriaga, L. (1981). Pedagogía. Buenos Aires: Losada.

Magendzo, A. (2008). Dilemas del currículum y la pedagogía. Santiago de Chile: LOM Editores.

Martínez-Otero, V. (2003). Proyección educativa de la inteligencia afectiva. Revista Complutense de Educación, 14(1), 57-82. Recuperado de http://revistas.ucm.es/index.php/RCED/article/ viewFile/RCED0303120057A/16471

Monereo, C. \& Solé, I. (1999). El asesoramiento psicopedagógico: una perspectiva profesional y constructivista. Madrid: Alianza Editorial.

Ossa, C. (2012). El rol del Psicólogo Educacional: la transición desde el paradigma de la simplicidad al paradigma de la complejidad. Revista Pequén, 1(1), 72-82. Recuperado de http://www.ubiobio. $\mathrm{cl} /$ miweb/webfile/media/265/el\%20rol\%20del\%20psicologo\%20 educacional.pdf

Roheim, G. (1943). The origin and function of culture. New York: Nervous and Mental Disease Monographs.

Sáez, R. (2006). La educación intercultural. Revista de Educación, 339(1), 859-881. Recuperado de http://www.revistaeducacion. mec.es/re339/re339a37.pdf

Stolp, S. (1994). Liderazgo para la cultura escolar. Clearinghouse on Educational Policy and Management. Recuperado de http:// www.ericdigests.org/2003-3/escolar.htm

Touriñán, J. (2011). Intervención educativa, intervención pedagógica y educación: la mirada pedagógica. Revista portuguesa de pedagogía, 283-307. Recuperado de iduc.uc.pt/index.php/rppedagogia/ article/download/1323/771

Vidal, J. (2007). Aportes del Psicólogo educacional a los establecimientos educativos. Santiago, Chile: Universidad de Chile. Recuperado de http://www.opech.cl/bibliografico/calidad_equidad/ aporte_psi_educ_centros_educativos.pdf

Vigotsky, L. (1979). The development of the higher forms of attention in childhood. Soviet Psychology, 23, 1-20. doi:10.2753/ RPO1061-0405180167 
